\title{
Is the Truth in Your Words? Distinguishing Children's Deceptive and Truthful Statements
}

\author{
Shanna Mary Williams, ${ }^{1}$ Victoria Talwar, ${ }^{1}$ R. C. L. Lindsay, ${ }^{2}$ Nicholas Bala, ${ }^{2}$ and Kang Lee ${ }^{3}$ \\ ${ }^{1}$ McGill University, Montreal, QC, Canada H3A OG4 \\ ${ }^{2}$ Queen's University, Kingston, ON, Canada K7L 3N6 \\ ${ }^{3}$ University of Toronto, Toronto, ON, Canada M5S 2J7
}

Correspondence should be addressed to Shanna Mary Williams; shanna.williams@mail.mcgill.ca

Received 24 June 2013; Accepted 21 October 2013; Published 22 January 2014

Academic Editor: Pär Anders Granhag

Copyright (c) 2014 Shanna Mary Williams et al. This is an open access article distributed under the Creative Commons Attribution License, which permits unrestricted use, distribution, and reproduction in any medium, provided the original work is properly cited.

Children's $(N=48)$ and adults' $(N=28)$ truthful and deceptive statements were compared using a linguistics-based computer software program. Children (4 to 7 years of age) and adults (18 to 25 years of age) participated in a mock courtroom experiment, in which they were asked to recount either a true or fabricated event. Testimonies were then analyzed using Linguistic Inquiry and Word Count Software (LIWC; Pennebaker et al. 2007). This software has been previously used to detect adults' deceptive statements (e.g., Bond and Lee, 2005). To date, no research has used this method on children's narratives, nor has this software been used to compare those narratives to adult counterparts. Markers generated through the LIWC program achieved detection rates of $72.40 \%$ for samples of both children's and adults' narratives combined. In contrast, adult laypersons' $(N=48)$ detection rates, for the same narratives (i.e., both children and adults) were close to chance. More specifically, detection rates were above chance for truth $(65.00 \%)$ and below chance for lies $(45.00 \%)$. Thus, the linguistic profile provided through LIWC yielded greater accuracy for evaluating the veracity of children's and adults' narratives compared to adult laypersons' detection accuracy.

\section{Introduction}

In the last three decades, greater numbers of children have testified in court $[1,2]$, which has led to an increased interest in research on the reliability and credibility of child witnesses $[3,4]$. To date child witness researchers have focused on children's testimonial capacities, reliability, and suggestibility, as well as jurors' perceptions and biases (e.g., [5-9]). Children are capable of giving highly accurate accounts and can make competent witnesses (e.g., $[10,11])$. However, relatively few studies have focused on methods of assessing the truthfulness of child witness testimonies [12].

Children's honesty is a debated issue in the justice system, as some have raised concerns that children may be easily coached in giving fabricated reports of abuse or in falsely recanting true allegations of abuse [13-16]. As children can be coached to tell false stories (e.g., $[2,17,18]$ ), research is needed to examine reliable methods for adults to accurately distinguish between children's truthful and deceptive statements.
The aim of the current study is to investigate the effectiveness of a computer-based software program, Linguistic Inquiry Word Count (LIWC), in distinguishing children's fabricated and truthful narratives. In addition, the computer-based analysis of child testimony was compared to computerbased analysis of adult testimony. Adult laypersons' deception detection accuracy was also conducted on the children's testimony, in order to determine the effectiveness of the software compared to adult laypersons' judgments.

\section{Development of Lie-Telling}

Children begin to tell lies around 3 years of age $[19,20]$. However, in order to be successful liars and to avoid detection, children must learn to monitor their nonverbal expressive behaviors. Even preschool children are able to successfully regulate their nonverbal behaviors in order to appear honest, and this ability improves with age [19-21]. 
In addition to regulating nonverbal expressive behavior, the successful lie-teller must also monitor their verbal statements in order to avoid inconsistencies in their false statements. Children's ability to maintain their lies and avoid detection has been shown to increase with age [20, 22-25]. Specifically, young children often have difficulty maintaining consistency throughout false statements and as a result incriminate themselves by leaking critical information about their own deceit $[20,21,24]$. As children grow older (especially after the age of 6 years), their ability to elaborate on lies and to maintain consistency across false statements increases [25].

In many studies examining children's lie-telling, narratives have been brief and limited to one or two statements about their own behaviors (e.g., [2, 21, 23]). When testifying in court, children are not typically asked to respond to brief questions but rather are required to provide many details of events that involve both themselves and others. Thus, in terms of forensic significance, it is more ecologically relevant to investigate children's abilities to generate free truthful and fabricated narratives about events involving themselves and others. Although it may be difficult to obtain long narratives from young children, free, uninterrupted, spontaneous narratives will provide researchers with a more ecologically valid measure of children's abilities.

\section{Assessing Children's True and Fabricated Reports}

Traditionally, research has reported that adults are able to detect children's lies at chance or slightly above chance levels (e.g., [26-28]). The few studies that reflect laypersons' abilities to detect children's lies do little to suggest that detection occurs at forensically useful levels. For example, Strömwall and Granhag [29] found that adults were better than chance at differentiating between children's paired truthful and false statements; however, adult performance was only slightly above chance levels (59.00\% accuracy). As adults are generally unable to see through children's deceptive strategies [30], more systematic methods of analyzing deceit have been developed, such as Reality Monitoring (RM) or CriteriaBased Content Analysis (CBCA).

Criteria-Based Content Analysis evaluates statements based on 19 criteria (e.g., logical structure and spontaneous corrections) with the presence of each criterion within a statement increasing the likelihood of the statement being genuine [31]. This procedure has been found to correctly categorize true reports $65.00 \%$ to $90.00 \%$ of the time [32]. Reality Monitoring (RM) has also been developed in an attempt to correctly classify truths and lies, with some studies showing it to be more effective than Criteria-Based Content Analysis [32]. The primary assumption of RM is that true memories are qualitatively different from false stories [33]. When an individual has experienced an event, they are more likely to recall this event from a perceptual basis. Therefore, Reality Monitoring is based on the principle that true statements will contain more sensory, contextual, and temporal information, while false stories will contain more references to cognitive mechanisms. Masip et al. [34] performed a meta-analysis of the deception detection findings using RM and concluded that, by using this technique, veracity of statements can be correctly classified at rates between $64.00 \%$ and $85.00 \%$.

While both of these methods have been used successfully to detect true, suggested, and false reports, there are some limitations to using these techniques with young children. Younger children's reports tend to contain fewer details resulting in shorter statements $[32,35,36]$. On average, both $\mathrm{RM}$ and CBCA can be used to discriminate between truthful and deceptive statements, when the statements themselves are lengthy [32]. Furthermore, the use of CBCA requires extensive training, despite the advantage of this method being designed specifically to analyze children's statements [37]. In contrast, RM requires less exhaustive training. However, both methods require instruction beyond that of the typical approaches used by investigators (e.g., police officers, lawyers, and judges: $[36,38])$. In response to the lengthy manual coding required for both RM and $\mathrm{CBCA}$, researchers have recently used advances in technology to develop computerbased software to analyze the veracity of adult statements $[39,40]$.

One such development in the field is the Linguistic Inquiry Word Count (LIWC) computer software program. This program detects semantic patterns of speech by analyzing text and calculating the frequencies of word use in relation to the total word count [41]. Semantic analysis using LIWC has been applied to adult accounts of true and false reports. Researchers have suggested that the advantages of this system of deception detection compared to other methods are that it is efficient, fast, and objective [40].

\section{Language-Based Analysis of Deception}

Researchers have employed the Linguistic Inquiry Word Count Software to analyze adults' truthful and deceptive statements [39, 42-44]. The LIWC program enables researchers to analyze the content of a statement based on the language of a written narrative. The LIWC analyzes words within a narrative and compares them to over 2000 words within a stored dictionary. Each word within a narrative is represented as a percentage of the text and can be identified as belonging to a larger dimension of language. Each dimension within the program's dictionary represents a subset of linguistic properties: (1) standard language, (2) psychological processes (e.g., metacognition, emotions, sensory, and perceptual), (3) relativity (e.g., space and time), and (4) personal concerns (e.g., occupation, leisure, and physical states) [41].

Using this dictionary, Newman et al. [40] found that deceptive statements were characterized by fewer first-person singular pronouns (e.g., I, me, my), fewer third person pronouns (e.g., he, she, they), more negative emotion words (e.g., hate, anger, enemy), fewer exclusive words (e.g., but, except, without), and more motion words (e.g., walk, move, go). It has been suggested that this linguistic pattern decreases cognitive demands, allowing lie-tellers to maintain consistency in their accounts and to simultaneously separate themselves from the lie $[39,40]$. For instance, lie-tellers may make fewer selfreferences because they wish to separate or "disassociate" themselves from the act of deception [45]. In contrast, 
when individuals are being truthful they are discussing an event that they have experienced, which is reflected in an "ownership" of the given narrative and results in more selfreferences [46]. Liars may also feel more guilt, either because of their lie or because of the topic they are lying about, which may lead them to express more negative emotion [47]. In addition to feelings of guilt and a desire to distance themselves from their lie, lie-tellers may experience a greater cognitive load as they try to keep their lie consistent and try to avoid detection. Lie-telling requires greater use of cognitive resources (compared to truth-telling), which leads deceivers to create less complex stories. Newman et al. [40] suggested that the use of exclusive words such as "but," "except," and "without" reflect greater cognitive complexity and creates narratives that are more precise in nature. Thus, lie-tellers can be expected to have fewer exclusive terms in an effort to reduce complexity and to be vague. This is consistent with other research, which suggests that deception requires more cognitive effort and higher processing abilities than telling the truth $[47,48]$.

In another study, Bond and Lee [39] used LIWC to examine prisoners' true and deceptive statements. Similar to Newman et al. [40], they found that prisoners' lies contained fewer self-references, other-references, and exclusive words, while having more negative emotion and motion words. They also compared the LIWC generated linguistic markers with RM markers to examine their accuracy in discriminating between true and fabricated reports. Bond and Lee [39] found that LIWC generated linguistic markers had an accuracy rate of $71.10 \%$ compared to RM markers' accuracy rate of $69.70 \%$. Researchers have concluded that the LIWC computer-based method can be an effective tool to increase the speed and objectivity of linguistic analyses of witness' statements [39].

While this method has the potential to assist in advancing knowledge about the composition of truthful and fabricated statements, the software's utility for classifying the veracity of children's reports remains untested. Although children do begin lie-telling at an early age and become proficient deceivers by elementary school age, there remain developmental differences in their false statements from their adult counterparts $[20,24]$. For example, children often lack semantic leakage control during follow-up questioning to a lie [23]. Thus, children often have difficulty providing plausible details, which are consistent with the initial false statements they make. Given the developmental differences in children's lie-telling behaviour and their cognitive and language abilities, it is unclear whether the LIWC linguistic markers will discriminate between children's truthful and fabricated reports.

\section{Current Study}

The current study examined both young children's (4 to 7 years of age) and adults' (18 to 25 years of age) true and fabricated narratives by using the LIWC software program to detect differences in the linguistic patterns of their reports. Both adult and child participants were instructed to recount either a true or fabricated story based on an event that either did or did not happen to them (e.g., going to a wedding or a sporting event). The age range for the child sample was selected because children's abilities to maintain their lies increases significantly between preschool years (4-5 years) and early elementary school years (6-7 years) [20, 2325]. Adult participants were recruited through a university undergraduate subject pool.

Previous researchers examining children's deception have primarily studied brief deceptive statements [21, 23]. In contrast, the current study examined children's longer narratives, which were elicited while children testified in a mock court. Furthermore, to date no study has directly examined these more detailed narratives in comparison to adult counter parts. Children and adults testified about the details of a truly experienced event involving themselves and others or an event that they had never actually experienced. All collected narratives were then transcribed and analyzed through the LIWC program. Linguistic markers of veracity were generated through the LIWC program and used to examine the differences and similarities between children's true and fabricated reports. These same linguistic markers were also used to analyze adults' fabricated and true reports.

Along with the veracity analysis provided through LIWC, "intuitive" abilities of adult laypersons to detect children's and adults' true and fabricated reports were also examined. Adult laypersons were asked to read transcriptions of both children's and adults' true and false statement. Furthermore, adult laypersons were asked to judge whether they believed the individual in the transcription was telling the truth or a lie. Finally, the judgments provided by adult laypersons were then compared to the deception rates obtained through the LIWC analysis.

\section{Methods}

6.1. Participants. Children $(N=48$; male $=29)$ between the ages of 4 and 7 years and adults between the ages of 18 and 25 years $(N=28$; male $=14)$ participated in a mock courtroom study. Of the child participants, half were $4-5$ years of age $(M=4.90, \mathrm{SD}=1.01)$ and half were $6-7$ years of age $\left(M_{\text {age }}=6.86, \mathrm{SD}=0.96\right)$. Half the children and adults told a lie and half told the truth. Participants were recruited through newspaper advertisements and flyers. Parental consent and child assent were obtained from all children prior to participating in the study.

Following the collection of truthful and deceptive statements, from both adult and child participants, adult laypersons were recruited to participate in a deception detection study. Adult laypersons, between the ages of 18 and $25\left(M_{\text {age }}=\right.$ 19.00 years, $\mathrm{SD}=2.04, N_{\text {males }}=21$ ), were recruited through a university undergraduate subject pool. Children's and adults' truthful and deceptive statements were viewed by these adult laypersons $(N=48)$.

\section{Procedure and Materials}

7.1. Children's True and Fabricated Reports. A total of 48 child interviews were used for the analyses. The interviews were collected as part of a mock courtroom study (see [49]). 
To obtain both truthful and deceptive statements, caregivers were asked to coach their child on one deceptive story and one truthful story. Parents were provided with a list of potential activities or events, which they could choose from (e.g., birthday parties and vacations). Prior to providing their statements, parents instructed their child to recount the story (15-minute session) in a mock courtroom to a research assistant. Children were randomly assigned to recount either a truthful or fabricated story condition. To ensure truthful stories were true and false stories were false both children and parents were asked after the narratives if the story was a truth or a lie. Stories were yoked so that there was a true and fabricated version of each type of story (i.e., a true story of attending a wedding and a fabricated story of attending a wedding). In both the truthful and deceptive conditions children were instructed to be as convincing as possible. All testimonies provided by the children within the mock courtroom were videotaped. During the testimony, children were asked to recount their true or fabricated stories, usually with only open-ended prompts from "counsel". The testimony portion of the interviews ranged in time from 1-10 minutes long.

7.2. Adult Statements Data Collection. A total of 28 adult interviews were used in the analysis. Similar to the children's interviews, adults were interviewed in a mock courtroom setting. Adults were instructed to tell a story, which was either fabricated or truthful. Adult participants were randomly assigned to either the truthful or deceptive stories condition and asked to tell one story. Similar to the children's stories, adult stories were also yoked (i.e., true and fabricated version of each type of story was collected). Following the story they were asked to state whether they told a truth or a lie.

7.3. Adult Deception Detection Data Collection. Adults $(N=$ 48) were asked to view videotaped testimonies of four children. Adults were individually placed at a computer where they viewed the video clips. They were asked to view the video clips (four each) and then rate each child as being either truthful or deceptive. They were not told the portion of truth-tellers and lie-tellers they would see in their four videos. Each adult rater viewed two truthful and two deceptive videos. Once all the videos had been viewed, participants were debriefed concerning the nature of the study.

7.4. Data Preparation for LIWC Analysis. Both adults' and children's narratives were transcribed and then analyzed using linguistic analysis software, which provides a description of text through a word-by-word count of text (LIWC; see for review [40]). The output of the program provides an analysis of word usage within the text and a percentage for each word used.

All interviews were transcribed word-for-word and then processed using the LIWC software. All transcriptions were saved individually into separate text files. The LIWC software provides 72 linguistic dimensions of speech, which can be further grouped into larger linguistic categories (e.g., Linguistic Processes, Psychological Processes, Personal Concerns, and Spoken Categories). These dimensions are represented within the output through percentage scores. All percentage scores were converted into $z$ scores. The conversion to $z$ scores allowed for comparisons between children within the lie and truth conditions. When using all 72 categories, LIWC accurately categorizes $80.00 \%$ of all words in a statement [41].

For the purposes of the present study, 29 categories were initially used for the LIWC analysis. In line with the procedure used by Newman et al. (2001), categories with low frequencies (less than $0.20 \%$ of the time), or categories left up to the discretion of the transcriber (e.g., assent, nonfluencies and fillers, such as "umm" or "uh") were excluded. In total, 20 variables were included in the subsequent analysis (see Table 1). The frequency of each category was obtained as a percentage for each story type (true or fabricated).

\section{Results}

Preliminary analysis revealed no significant gender differences for both adult and children samples on any of the LIWC categories. Thus, the data of both genders were combined for all subsequent analyses. Results will first be presented by examining the findings from the LIWC software program followed by the generated LIWC model's predictability to accurately discriminate true and fabricated reports (i.e., adults and children combined) and adults' detection abilities.

\subsection{LIWC Analysis}

8.1.1. Length of Stories. The length of each story, as indexed by word count, was investigated using a 2 (Veracity: true versus fabricated) $\times 3$ (Age Group: 4-5-year old, 6-7-year old; adults) Analysis of Variance (ANOVA) on mean word count scores. There was a significant Age Group difference, $F(2,75)=34.03, P<0.001, \eta^{2}=0.49$. Adults' stories were significantly longer $\left(M_{\text {adult }}=302.18, \mathrm{SD}=123.13\right)$ than children's stories $\left(M_{4-5}=94.47 ; \mathrm{SD}=72.64, M_{6-7}=126.93\right.$, $\mathrm{SD}=79.95, P<0.001)$. There was no significant difference between truthful and false stories. Overall, both child and adult participants' true $(M=190.61, \mathrm{SD}=114.61)$ and fabricated $(M=177.16, \mathrm{SD}=115.52)$ stories were similar in length.

To evaluate whether adults and children's true and fabricated reports differ in the types of words we used, a series of 2 (Veracity: true versus fabricated) ANOVAs were conducted for the 19 remaining LIWC categories. Results revealed significant differences between the narratives for eight linguistic variables: singular self-references (e.g., I, me, my), plural self-references (e.g., we, our, us), positive emotional words (e.g., happy, pretty, good), negative emotional words (e.g., references to anxiety, sadness, anger), cognitive processes terms (e.g., cause, know, ought), tentative terms (e.g., maybe, guess), spatial terms (e.g., down, in), and exclusive terms (e.g., but, without, exclude). These variables were applied to the analysis of the adults and children's narratives. Each variable will be discussed in turn.

8.1.2. Singular Self-References. The 2 (Veracity) $\times 3$ (Age Groups) ANOVA on children's and adults' use of first person 
TABLE 1: The mean frequency (standard deviation) of the Linguistic Inquiry Word Count categories by narrative type.

\begin{tabular}{|c|c|c|c|c|c|c|c|}
\hline \multirow{2}{*}{ Dimension } & \multirow{2}{*}{ Example } & \multicolumn{3}{|c|}{ Lie } & \multicolumn{3}{|c|}{ True } \\
\hline & & Younger & Older & Adults & Younger & Older & Adults \\
\hline Word count & & $78.56(48.55)$ & $142.53(96.72)$ & $300.53(126.45)$ & $112.6(88.59)$ & $110.21(55.77)$ & $304.57(109.45)$ \\
\hline Words per sentence & & $9.97(6.37)$ & $19.20(13.30)$ & $55.45(46.87)$ & $23.56(24.36)$ & $16.77(6.91)$ & $29.27(11.89)$ \\
\hline $\begin{array}{l}\text { Words longer than six } \\
\text { letters }\end{array}$ & & $11.20(6.78)$ & $7.54(2.82)$ & $10.92(2.37)$ & $8.63(5.14)$ & $8.87(3.86)$ & $10.66(1.98)$ \\
\hline Unique & & $60.85(12.73)$ & $55.75(14.18)$ & $46.16(6.03)$ & $54.37(20.60)$ & 57.97 (15.09) & $45.49(5.51)$ \\
\hline First person singular & I, my & $10.41(5.09)$ & $7.44(4.65)$ & $6.12(2.71)$ & $6.72(3.19)$ & $6.13(2.22)$ & $5.84(3.41)$ \\
\hline First person plural & We, us & $00.92(1.96)$ & $5.38(4.70)$ & $3.01(2.22)$ & $5.79(5.10)$ & $4.32(3.20)$ & $3.16(2.70)$ \\
\hline Total self-references & I, we & $10.22(5.31)$ & $11.56(4.97)$ & $9.13(2.40)$ & $12.06(3.75)$ & $10.46(3.31)$ & $9.00(2.24)$ \\
\hline Second person pronouns & You, your & $0.00(0.00)$ & $-0.67(1.22)$ & $0.57(0.65)$ & $0.53(1.69)$ & $0.60(1.43)$ & $0.37(0.58)$ \\
\hline Third person pronouns & She, their & $3.38(3.77)$ & $1.51(1.52)$ & $1.9(1.43)$ & $1.38(2.43)$ & $1.13(1.47)$ & $2.06(1.59)$ \\
\hline Positive emotions & Happy, pretty & $0.48(0.80)$ & $1.16(1.73)$ & $2.01(1.36)$ & $0.58(0.73)$ & $1.76(1.62)$ & $1.87(1.07)$ \\
\hline Negative emotions & Hate, enemy & $0.57(0.61)$ & $1.03(0.48)$ & $0.87(0.92)$ & $0.08(0.22)$ & $0.08(0.22)$ & $1.05(0.91)$ \\
\hline Cognitive processes & Know, ought & $3.8(2.62)$ & $2.17(1.44)$ & $5.92(1.91)$ & $1.89(1.84)$ & $2.82(1.89)$ & $5.63(2.14)$ \\
\hline Discrepancy terms & Should, would & $0.29(0.57)$ & $0.28(0.44)$ & $1.79(0.99)$ & $0.60(0.78)$ & $0.99(1.61)$ & $1.61(1.01)$ \\
\hline Tentative terms & Maybe, guess & $1.10(2.55)$ & $0.36(0.65)$ & $2.78(0.65)$ & $0.97(1.23)$ & $1.26(1.09)$ & $2.39(1.41)$ \\
\hline $\begin{array}{l}\text { Sensory/perceptual } \\
\text { processes }\end{array}$ & See, touch & $1.72(1.47)$ & $1.48(1.07)$ & $1.52(1.15)$ & $0.80(1.02)$ & $1.56(1.21)$ & $1.34(0.99)$ \\
\hline Time & Hour, day & $3.89(4.02)$ & $4.82(3.12)$ & $3.96(1.74)$ & $4.21(4.70)$ & $4.15(2.66)$ & $4.17(1.81)$ \\
\hline Space & Around, over & $3.43(3.70)$ & $4.50(2.50)$ & $4.29(1.25)$ & $6.79(5.19)$ & $5.79(3.23)$ & $4.26(1.60)$ \\
\hline Exclusive & But, without & $1.48(1.60)$ & $2.00(1.80)$ & $3.61(1.25)$ & $2.67(2.32)$ & $3.50(2.93)$ & $3.93(1.37)$ \\
\hline Motion & Walk, move & $3.12(2.14)$ & $4.26(2.97)$ & 3.39 (1.49) & $4.42(2.52)$ & $3.67(2.56)$ & $3.94(0.95)$ \\
\hline Certainty & Always, never & $0.24(0.53)$ & $0.53(0.73)$ & $2.75(1.51)$ & $0.26(0.62)$ & $0.53(0.80)$ & $2.47(1.00)$ \\
\hline
\end{tabular}

singular self-references revealed a main effect for Veracity, $F(1,75)=6.36, P=0.014, \eta^{2}=0.13$. Overall participants' (both children and adults) fabricated stories $(M=7.99$, $\mathrm{SD}=4.46)$ had more first person singular references than their true stories $(M=6.21, \mathrm{SD}=2.89)$. An interaction of Veracity by Age Group did approach significance, $F(2,75)=$ 2.77, $P=0.07, \eta^{2}=0.074$. Overall, young children used significantly more singular self-references in their fabricated stories $(M=10.41, \mathrm{SD}=5.09)$ compared to their true stories $(M=6.72, \mathrm{SD}=3.19)$ and compared to both older children and adults. Older children also used more self-references in their fabricated stories $(M=7.44, \mathrm{SD}=4.65)$ compared to their true stories $(M=6.13, \mathrm{SD}=2.22)$. Adults also used more self-references in their false stories $(M=6.12$, $\mathrm{SD}=2.71)$ than in their true stories $(M=5.84, \mathrm{SD}=3.41)$.

8.1.3. Plural Self-References. The 2 (Veracity) $\times 3$ (Age Groups) ANOVA on children's and adults' use of first person plural self-references revealed a main effect of Age Group, $F(2,75)=79.95, P<0.001, \eta^{2}=0.69$. Younger children used plural self-references less frequently $(M=3.45, \mathrm{SD}=$ 4.53) compared to older children $(M=4.87, \mathrm{SD}=4.01$, $P<0.05)$. There was also an interaction effect between Veracity and Age Group $F(2,75)=4.24, P=0.018, \eta^{2}=$ 0.11 . Younger children (4-5 years) had significantly fewer plural self-references for their fabricated stories $(M=.92$, $\mathrm{SD}=1.96)$ compared to their true stories $(M=5.79$, $\mathrm{SD}=5.10)$, while older children's true $(M=4.32, \mathrm{SD}=3.20)$ and fabricated stories $(M=5.38, \mathrm{SD}=4.70)$ had similar frequencies of plural self-references. Similar to older children, adults also had similar rates of plural self-references in both their true $(M=3.16, \mathrm{SD}=2.70)$ and fabricated stories $(M=3.01, \mathrm{SD}=2.22)$.

8.1.4. Positive Emotions. The 2 (Veracity) $\times 3$ (Age Groups) ANOVA on children's and adults' expressions of positive emotions revealed a main effect for Age Group, $F(2,75)=$ $23.38, P<0.001, \eta^{2}=.40$. Younger children $(M=0.48, \mathrm{SD}=$ $0.80)$ described fewer positive emotions in their fabricated stories than older children $(M=1.16, \mathrm{SD}=1.73, P<$ 0.05). Adults used the most positive emotions compared to all children $(M=2.01, \mathrm{SD}=1.36, P s<0.001)$.

8.1.5. Negative Emotions. The 2 (Veracity) $\times 3$ (Age Groups) ANOVA on children's expression of negative emotions revealed a main effect for Veracity, $F(1,75)=28.07, P<$ $0.001, \eta^{2}=0.29$. Both children's and adults' true stories $(M=0.11, \mathrm{SD}=0.21)$ described fewer negative emotions than their fabricated stories $(M=0.86, \mathrm{SD}=0.56)$. There was a significant interaction between Veracity and Age Group, $F(2,75)=6.47, P=0.03, \eta^{2}=0.16$. Young children described less negative emotions in their fabricated stories $(M=0.57, \mathrm{SD}=0.61)$ than older children $(M=1.03$, $\mathrm{SD}=0.48)$. In contrast to children, adults described negative emotions the least in their fabricated stories $(M=0.87$, $\mathrm{SD}=0.92)$. Children used fewer negative emotions in their 
true stories (younger children $M=0.08, \mathrm{SD}=0.22$; older children $M=0.08, \mathrm{SD}=0.22$; adults $M=1.05, \mathrm{SD}=0.91$ ).

8.1.6. Cognitive Processes. The 2 (Veracity) $\times 3$ (Age Groups) ANOVA on children's use of cognitive processes terms revealed a main effect of Age Group, $F(2,75)=22.05, P<$ $.001, \eta^{2}=0.39$. Adults $(M=5.79, \mathrm{SD}=2.01)$ used more cognitive processes than younger children $(M=2.79$, $\mathrm{SD}=2.63)$ and older children $(M=2.48, \mathrm{SD}=1.67)$. There was also a significant interaction between Veracity and Age Group, $F(1,75)=3.63, P=0.02, \eta^{2}=0.10$. Younger children had the most cognitive processes in their fabricated reports $(M=3.80, \mathrm{SD}=2.62)$ than true reports $(M=$ $1.89, \mathrm{SD}=1.84)$. Older children had similar rates in their fabricated $(M=2.17, \mathrm{SD}=1.44)$ and true reports $(M=$ $2.82, \mathrm{SD}=1.89)$. Adults had the most number of cognitive processes in both fabricated $(M=5.92, \mathrm{SD}=1.91)$ and true reports $(M=5.63, \mathrm{SD}=2.14)$.

8.1.7. Tentative Terms. The 2 (Veracity) $\times 3$ (Age Groups) ANOVA on tentative terms revealed a main effect of Age Group, $F(2,75)=3.633, P=0.032, \eta^{2}=0.09$. Younger children $(M=1.08, \mathrm{SD}=1.91)$ were not significantly different from older children $(M=0.80, \mathrm{SD}=0.89)$ but did have significantly less tentative terms than adults $(M=2.61$, $\mathrm{SD}=.91)$.

8.1.8. Spatial Terms. The 2 (Veracity) $\times 3$ (Age Groups) ANOVA on children and adults' use of spatial terms revealed a main effect for Veracity, $F(2,75)=5.16, P=0.026, \eta^{2}=$ 0.07. Participants' true stories $(M=6.29, \mathrm{SD}=4.08)$ had more spatial terms than their fabricated stories $(M=3.97$, $\mathrm{SD}=2.97)$. There was also a main effect of Age Group, $F(2,75)=25.66, P<0.001, \eta^{2}=0.42$. Younger children $(M=5.19, \mathrm{SD}=4.47)$ and older children $(M=5.12$, $\mathrm{SD}=2.9)$ used more spatial terms than adults $(M=4.27$, $\mathrm{SD}=1.35, P>.05)$.

8.1.9. Exclusive Terms. The 2 (Veracity) $\times 3$ (Age Groups) ANOVA on children's and adults' use of exclusive terms revealed a main effect for Age Group, $F(2,75)=61.4, P<$ $0.001, \eta^{2}=0.637$. Adults used significantly more exclusive terms in their stories $(M=3.78, \mathrm{SD}=1.33)$ compared to both young children $(M=2.10, \mathrm{SD}=2.05)$ and older children $(M=2.90, \mathrm{SD}=2.48)$.

8.2. Discriminant Analysis of Statements. As ANOVAs revealed significant linguistic differences between true and false reports, a stepwise discriminant analysis using Wilks' Lambda method was conducted to determine whether the linguistic trends could predict the veracity of both children's and adults' statements combined. The LIWC generated model (using the LIWC verbal markers) was examined using stepwise discriminant analyses. In all analyses the veracity of the statement was used as the classifying variable.

8.2.1. LIWC Generated Analysis. In order to examine whether the linguistic trends found between the current study's true and fabricated reports predicted veracity, 5 dependent variables were entered into the analysis: (singular self-references, plural self-references, negative emotional words, cognitive processes, and spatial terms). The overall Wilks' lambda was significant, Wilks' $\Lambda=0.75, \chi^{2}(1, N=76)=$ 23.13, $P<0.001$, indicating that participants' true and false narratives were successfully differentiated well above chance levels (72.40\% of the time). Classification rates for lie statements were $57.94 \%$ and for true statements they were $86.80 \%$. The beta coefficient for first-person singular references $(\beta=00.91)$ was statistically significant, with more self-references significantly predicting that the story is false, $F(1,46)=4.33, P=0.04$. The beta coefficient for negative emotions $(\beta=0.51)$ was also statistically significant, with more negative emotions significantly predicting that the story is false, $F(1,46)=45.57, P<0.001$. The beta coefficient for spatial references $(\beta=0.92)$ was statistically significant, with more spatial references significantly predicting that the story is true, $F(1,46)=4.15, P=0.04$. All other coefficients were nonsignificant.

\subsection{Adult Laypersons' Ratings of True and False Reports}

8.3.1. Overall Accuracy Rates. Accuracy rates were calculated for each adult layperson participant by averaging their accuracy across both true and false stories. Overall accuracy rates ranged from 0.28 to $0.68(M=0.49, \mathrm{SD}=0.07)$. A one-sample $t$-test comparing accuracy rates to chance (0.50) indicated that the mean overall accuracy rate was not significantly different from chance, $t(95)=-0.2$, n.s.

Further one-sample $t$-tests compared the accuracy rates for truthful and fabricated stories separately to chance levels. Adult accuracy rates for true stories were found to be above chance levels $(M=0.65, \mathrm{SD}=0.15), t(95)=2.85, P>0.05$, while false stories were significantly below chance levels $(M=$ $0.45, \mathrm{SD}=0.14), t(95)=3.30, P<0.05$. Thus, it appears that adult participants may have been biased to respond "true" when reading the stories.

\section{Discussion}

The present study examined children's and adults' linguistic markers for deception using Linguistic Inquiry Word Count, a computer based linguistic software. Results of the study revealed significant linguistic differences between true and false narratives, as well as between the different age groups within the sample.

9.1. Differences between True and Fabricated Reports. Overall, when children's and adults' statements were examined together, the LIWC program had a high success rate $(72.40 \%)$. When linguistic markers, generated through the LIWC analysis were analyzed closely, some differences between adults' and children's statements were found. Specifically young children's deceptive narratives contained significantly more first person singular words than their truthful narratives. Children's greater use of first person singular words, during deception, may be related to their egocentric nature observed by researchers during this stage in development $[50,51]$. 
Perhaps young children's use of egocentric speech is more frequent when creating imaginary scenarios (i.e., lie-telling) and as a result they are more likely to use first person singular references in their fabricated reports. The difference between children's and adults' use of first person singular references is unexpected, and as a result, more research examining this factor in relation to determining the veracity of children's statements is required. Consistent with previous research, our results do suggest that the frequency of first person singular references can distinguish true and fabricated reports. However, more research is needed to examine the direction of that effect and its relation to age.

Consistent with adult data, participants' true narratives contained fewer negative emotions than their deceptive narratives. Thus, like adults from previous research, children may experience feelings of guilt when deceiving, which results in greater use of negative emotion words when lying [40]. The sample of adults within the current study did use less negative emotions within their false statements compared to their truthful statements, although a statistical difference was not found.

Consistent with previous research using RM theory, participants' true narratives had significantly more spatial terms than their deceptive narratives. Bond and Lee [39] found greater use of spatial words for adults' true reports when they used LIWC to analyze narratives. A significant age effect was also found with children using more spatial terms in their true statements compared to adult counterparts.

Also consistent with RM theory, younger children's false reports had more cognitive processes than their true reports. However, this was not the case for older children. Finally, adults used the greatest amount of cognitive processes in their false statements, compared to both younger age groups.

Taken together, most of the findings in the present study are consistent with previous research, which suggests that with age children's abilities to maintain their lies beyond 1 or 2 statements improves (e.g., [20, 24, 25]). Overall, these results suggest that children's verbal markers of deception may be a result of their attempts to decrease cognitive load and children's age (construction of fabricated reports improves with age). Furthermore, compared to adults, children may use different strategies when creating fabricated reports and these strategies may change over the course of development.

9.2. Linguistic Markers of Deception Derived from LIWC Analysis. The research of Bond and Lee [39] supports the use of LIWC in the categorization of true and fabricated reports. Within the current study, when both adults' and children's statements were examined using a discriminant analysis, support was found for the use of LIWC in the categorization across ages. Compared to LIWC generated markers, adult detectors were only capable of accurately identifying the veracity of statements at chance levels (49\%). These findings as well as the Bond and Lee [39] results suggest LIWC generated analysis is more accurate than layperson detection. Hence, the findings of the current study provide support for the use of the LIWC program in generating linguistic markers for the purposes of judging the veracity of children's statements.
The current research supports the use of LIWC in categorizing both adults' and children's true and deceptive statements. Although methods such as Reality Monitoring have yielded detection accuracy rates above chance, the drawback of this approach is that it requires longer training than LIWC and continues to be subject to the accuracy of the individual raters [36]. Conversely, analysis using LIWC does not require lengthy training and is relatively inexpensive to conduct on transcribed narratives. Furthermore, the performance of human raters using the RM theoretical framework can be subject to variability of interrater reliability (see [38, 47]). Conversely, LIWC is not subject to the variability of human raters, therefore providing consistent categorization of linguistic markers across narratives. Thus, using LIWC computer software analysis may be a more effective method of assessment than other methods of analyses (e.g., RM) as LIWC allows for quicker scrutiny of reports and greater objectivity [44]. LIWC software analysis may be a viable alternative technique for classifying true and fabricated reports.

9.3. Limitations. There are several caveats that need to be considered regarding the current study and the use LIWC for deception detection. First, children's development of language was not adequately assessed. As previously noted, the ability to describe an event develops with age. Young children's narratives are shorter and less detail rich. Future studies should provide an analysis of children's verbal abilities as a potential covariate. A particularly important issue will be the determination of the age at which the direction of effects changes from the pattern observed for children to that of adults changes. To further support this finding, an additional study should be completed with a larger sample size. The current study consisted of 48 child participants who were split between truthful and false statements; an increase in sample size would validate and support the current findings.

Future research should directly compare and measure the accuracy of the LIWC method to other approaches discussed in this paper (i.e., CBCA and Reality Monitoring). It is important to note that the current study is the first attempt to measure children's narratives using the LIWC method and thus the researchers were limited in their capacity to directly measure and compare the LIWC method to other deception detection techniques. Future studies should directly examine the accuracy of approaches such as RM and CBCA to the LIWC approach.

\section{Conclusion}

The ability to effectively detect children's deceptive statements has potential implications for the justice system professionals (e.g., police, lawyers, child protection workers, and judges). As children become increasingly called upon to testify in court, it is important to determine effective methods of analyzing their statements. LIWC computer software is a potential tool for investigators because it quickly and efficiently analyzes such reports. The present study is the first of its kind to provide an analysis of children's narratives using this software. LIWC effectively discriminates between 
children's true and fabricated reports. However, it should be noted that this classification tool is still in its initial phases of development and testing; therefore, it has yet to be applied to a wide variety of settings. Additional research and replication are needed to examine its utility across a variety of situations and ages. The current study provides a starting point for further developing linguistic analysis software, which can be used to determine the veracity of children's statements.

\section{Conflict of Interests}

The authors declare that there is no conflict of interests regarding the publication of this paper.

\section{References}

[1] G. M. Davis, "Coping with suggestion and deception in children's accounts," in The Detection of Deception in Forensic Contexts, P. A. Granhag and L. A. Stromwall, Eds., pp. 148-171, Cambridge University Press, England, UK, 2004.

[2] V. Talwar, K. Lee, N. Bala, and R. C. L. Lindsay, "Children's lietelling to conceal a parent's transgression: legal implications," Law and Human Behavior, vol. 28, no. 4, pp. 411-435, 2004.

[3] N. Bala, K. Lee, R. C. L. Lindsay, and V. Talwar, "The competency of children to testify: psychological research informing canadian law reform," International Journal of Children's Rights, vol. 18, no. 1, pp. 53-77, 2010.

[4] S. J. Ceci, S. Kulkofsky, J. Z. Klemfuss, C. D. Sweeney, and M. Bruck, "Unwarranted assumptions about children's testimonial accuracy," Annual Review of Clinical Psychology, vol. 3, pp. 311328, 2007.

[5] B. L. Bottoms and G. S. Goodman, "Perceptions of children's credibility in sexual assault cases," Journal of Applied Social Psychology, vol. 24, pp. 702-732, 1994.

[6] S. J. Ceci and M. Bruck, "Suggestibility of the child witness: a historical review and synthesis," Psychological Bulletin, vol. 113, no. 3, pp. 403-439, 1993.

[7] M. Bruck, S. J. Ceci, and H. Hembrooke, "Reliability and credibility of young children's reports: from research to policy and practice," American Psychologist, vol. 53, no. 2, pp. 136-151, 1998.

[8] M. R. McCauley and J. F. Parker, "When will a child be believed? The impact of the victim's age and juror's gender on children's credibility and verdict in a sexual-abuse case," Child Abuse and Neglect, vol. 25, no. 4, pp. 523-539, 2001.

[9] D. F. Ross, D. Dunning, M. P. Toglia, and S. J. Ceci, "The child in the Eyes of the jury. Assessing mock jurors' perceptions of the child witness," Law and Human Behavior, vol. 14, no. 1, pp. 5-23, 1990.

[10] M. Bruck and S. J. Ceci, “The suggestibility of children's memory," Annual Review of Psychology, vol. 50, pp. 419-439, 1999.

[11] J. A. Quas, G. S. Goodman, S. Ghetti, and A. D. Redlich, "Questioning the child witness: what can we conclude from the research thus far?" Trauma, Violence, and Abuse, vol. 1, no. 3, pp. 223-249, 2000.

[12] M. C. Tye, S. L. Amato, C. R. Honts, M. K. Kevitt, and D. Peters, "The willingness of children to lie and the assessment of credibility in an ecologically relevant laboratory setting," Applied Developmental Science, vol. 3, no. 2, pp. 92-109, 1999.
[13] N. Bala and J. Schuman, "Allegations of sexual abuse when parents have separated," Canadian Family Law Quarterly, vol. 17, pp. 191-243, 1999.

[14] M. Brennan, "The battle for credibility-themes in the cross examination of child victim witnesses," International Journal for the Semiotics of Law, vol. 7, no. 1, pp. 51-73, 1994.

[15] P. Ekman, "The argument and evidence about universals in facial expressions of emotion," in Handbook of Social Psychophysiology, H. Wagner and A. Manstead, Eds., John Wiley \& Sons, Chichester, UK, 1989.

[16] J. J. Haugaard and N. D. Reppucci, "Children and the truth," in Cognitive and Social Factors in Early Deception, S. J. Ceci, M. D. Leichtman, and M. E. Putnick, Eds., pp. 29-45, Erlbaum, Hillsdale, NJ, USA, 1992.

[17] T. D. Lyon, L. C. Malloy, J. A. Quas, and V. A. Talwar, "Coaching, truth induction, and young maltreated children's false allegations and false denials," Child Development, vol. 79, no. 4, pp. 914-929, 2008.

[18] H. K. Orcutt, G. S. Goodman, A. E. Tobey, J. M. BattermanFaunce, and S. Thomas, "Detecting deception in children's testimony: factfinders' abilities to reach the truth in open court and closed-circuit trials," Law and Human Behavior, vol. 25, no. 4, pp. 339-372, 2001.

[19] M. Lewis, C. Stanger, and M. W. Sullivan, "Deception in 3-yearolds," Developmental Psychology, vol. 25, no. 3, pp. 439-443, 1989.

[20] V. Talwar and K. Lee, "Development of lying to conceal a transgression: children's control of expressive behaviour during verbal deception," International Journal of Behavioral Development, vol. 26, no. 5, pp. 436-444, 2002.

[21] V. Talwar, S. M. Murphy, and K. Lee, "White lie-telling in children for politeness purposes," International Journal of Behavioral Development, vol. 31, no. 1, pp. 1-11, 2007.

[22] A. Polak and P. L. Harris, "Deception by young children following noncompliance," Developmental Psychology, vol. 35, no. 2, pp. 561-568, 1999.

[23] V. Talwar, H. M. Gordon, and K. Lee, "Lying in the elementary school years: verbal deception and its relation to second-order belief understanding," Developmental Psychology, vol. 43, no. 3, pp. 804-810, 2007.

[24] V. Talwar and K. Lee, "Emergence of white-lie telling in children between 3 and 7 years of age," Merrill-Palmer Quarterly, vol. 48, no. 2, pp. 160-181, 2002.

[25] V. Talwar and K. Lee, "Social and cognitive correlates of children's lying behavior," Child Development, vol. 79, no. 4, pp. 866-881, 2008.

[26] K. Chahal and T. Cassidy, "Deception and its detection in children: a study of adult accuracy," Psychology, Crime and Law, vol. 1, no. 3, pp. 237-245, 1995.

[27] A.-M. Leach, V. Talwar, K. Lee, N. Bala, and R. C. L. Lindsay, “"Intuitive" lie detection of children's deception by law enforcement officials and university students," Law and Human Behavior, vol. 28, no. 6, pp. 661-685, 2004.

[28] L. H. Westcott, G. M. Davies, and R. B. Clifford, "Adults' perceptions of children's videotaped truthful and deceptive statements," Children \& Society, vol. 5, pp. 123-135, 1991.

[29] L. A. Strömwall and P. A. Granhag, "Children's repeated lies and truths: effects on adults' judgments and reality monitoring scores," Psychiatry, Psychology and Law, vol. 12, no. 2, pp. 345356, 2005. 
[30] L. A. Strömwall and P. A. Granhag, "Detecting deceit in Pairs of children," Journal of Applied Social Psychology, vol. 37, no. 6, pp. 1285-1304, 2007.

[31] A. Vrij, W. Kneller, and S. Mann, "The effect of informing liars about Criteria-Based Content Analysis on their ability to deceive CBCA-raters," Legal and Criminological Psychology, vol. 5, no. 1, pp. 57-70, 2000.

[32] A. Vrij, "Criteria-based content analysis: a qualitative review of the first 37 studies," Psychology, Public Policy, and Law, vol. 11, no. 1, pp. 3-41, 2005.

[33] M. K. Johnson, S. Hashtroudi, and D. Stephen Lindsay, "Source monitoring," Psychological Bulletin, vol. 114, no. 1, pp. 3-28, 1993.

[34] J. Masip, S. L. Sporer, E. Garrido, and C. Herrero, "The detection of deception with the reality monitoring approach: a review of the empirical evidence," Psychology, Crime and Law, vol. 11, no. 1, pp. 99-122, 2005.

[35] G. S. Goodman and R. S. Reed, "Age differences in eyewitness testimony," Law and Human Behavior, vol. 10, no. 4, pp. 317-332, 1986.

[36] A. Vrij, L. Akehurst, S. Soukara, and R. Bull, "Detecting deceit via analyses of verbal and nonverbal behavior in children and adults," Human Communication Research, vol. 30, no. 1, pp. 841, 2004.

[37] L. Akehurst, R. Bull, A. Vrij, and G. Köhnken, "The effects of training professional groups and lay persons to use criteriabased content analysis to detect deception," Applied Cognitive Psychology, vol. 18, no. 7, pp. 877-889, 2004.

[38] S. L. Sporer, "The less travelled road to truth: verbal cues in deception detection in accounts of fabricated and selfexperienced events," Applied Cognitive Psychology, vol. 11, no. 5, pp. 373-397, 1997.

[39] G. D. Bond and A. Y. Lee, "Language of lies in prison: linguistic classification of prisoners' truthful and deceptive natural language," Applied Cognitive Psychology, vol. 19, no. 3, pp. 313-329, 2005.

[40] M. L. Newman, J. W. Pennebaker, D. S. Berry, and J. M. Richards, "Lying words: predicting deception from linguistic styles," Personality and Social Psychology Bulletin, vol. 29, no. 5, pp. 665-675, 2003.

[41] J. W. Pennebaker, M. E. Francis, and R. J. Booth, Linguistic Inquiry and Word Count (LIWC): LIWC, 2001, Erlbaum, Mahwah, NJ, USA, 2001.

[42] E. Krackow, "Narratives distinguish experienced from imagined childhood events," The American Journal of Psychology, vol. 123, no. 1, pp. 71-80, 2010.

[43] J. W. Pennebaker, R. J. Booth, and M. E. Francis, Linguistic Inquiry and Word Count (LIWC2007): A Text Analysis Program, LIWC.net, Austin, Tex, USA, 2007.

[44] A. Vrij, S. Mann, S. Kristen, and R. P. Fisher, "Cues to deception and ability to detect lies as a function of police interview styles," Law and Human Behavior, vol. 31, no. 5, pp. 499-518, 2007.

[45] L. M. Knapp, P. R. Hart, and S. H. Dennis, "An exploration of deception as a communication construct," Human Communication Research, vol. 1, pp. 15-29, 1974.

[46] P. Grice, Studies in the Way of Words, Harvard University Press, Cambridge, Mass, USA, 1989.

[47] A. Vrij, Detecting Lies and Deceit: The Psychology of Lying and Its Implications for Professional Practice, John Wiley \& Sons, Chichester, UK, 2000.

[48] S. L. Sporer and B. Schwandt, "Paraverbal indicators of deception: a meta-analytic synthesis," Applied Cognitive Psychology, vol. 20, no. 4, pp. 421-446, 2006.
[49] V. Talwar, K. Lee, N. Bala, and R. C. L. Lindsay, "Adults' judgments of child witness credibility and veracity," Law and Human Behavior, vol. 30, pp. 561-570, 2006.

[50] N. Epley, C. K. Morewedge, and B. Keysar, "Perspective taking in children and adults: equivalent egocentrism but differential correction," Journal of Experimental Social Psychology, vol. 40, no. 6, pp. 760-768, 2004.

[51] J. H. Flavell, P. T. Botkin, C. L. Fry, J. C. Wright, and P. E. Jarvis, "The development of communication and role-taking skills in children," 1968. 

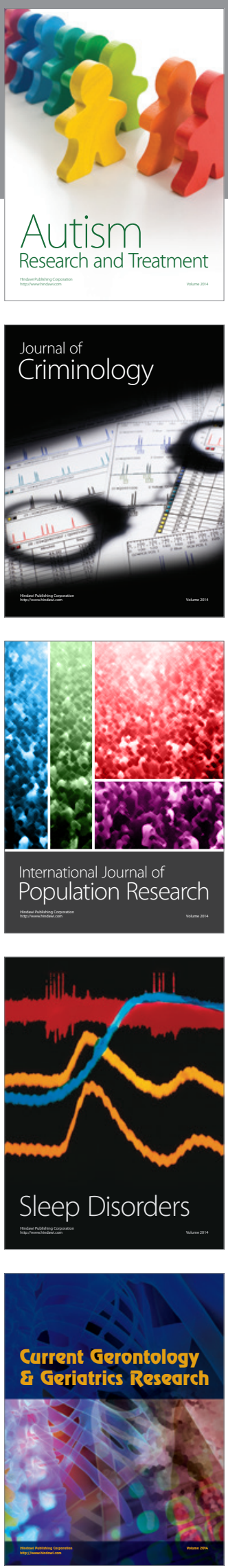
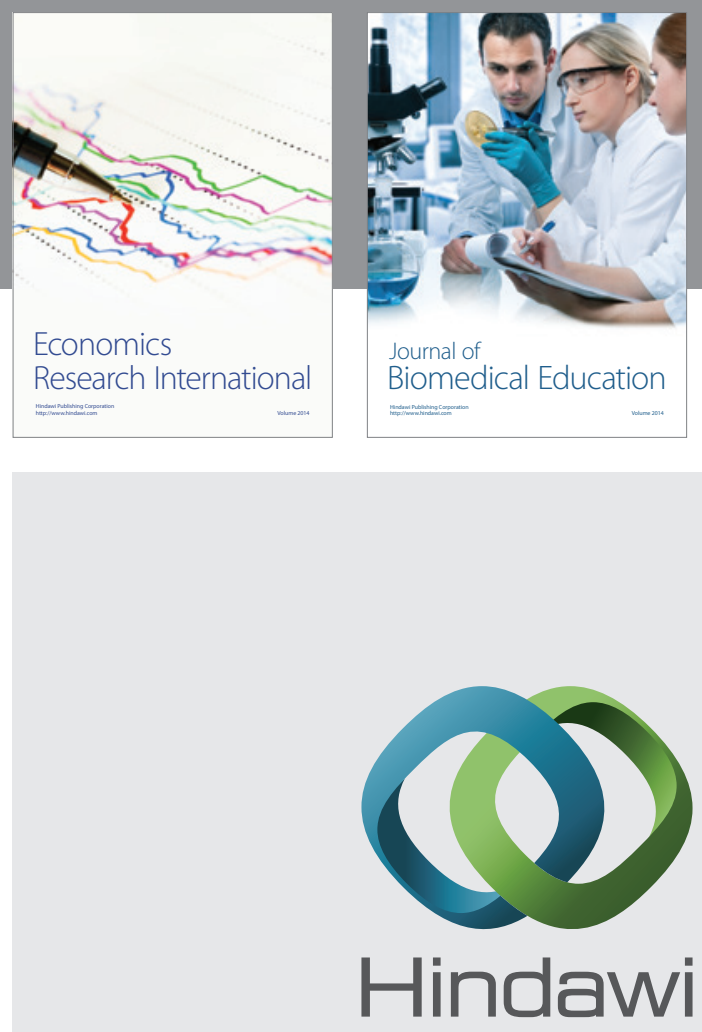

Submit your manuscripts at

http://www.hindawi.com
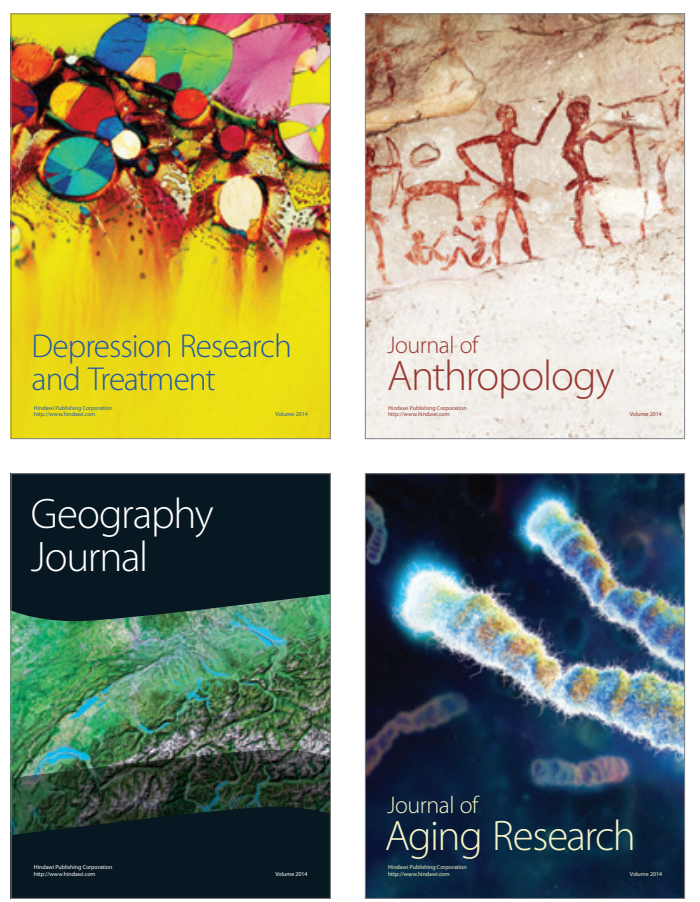
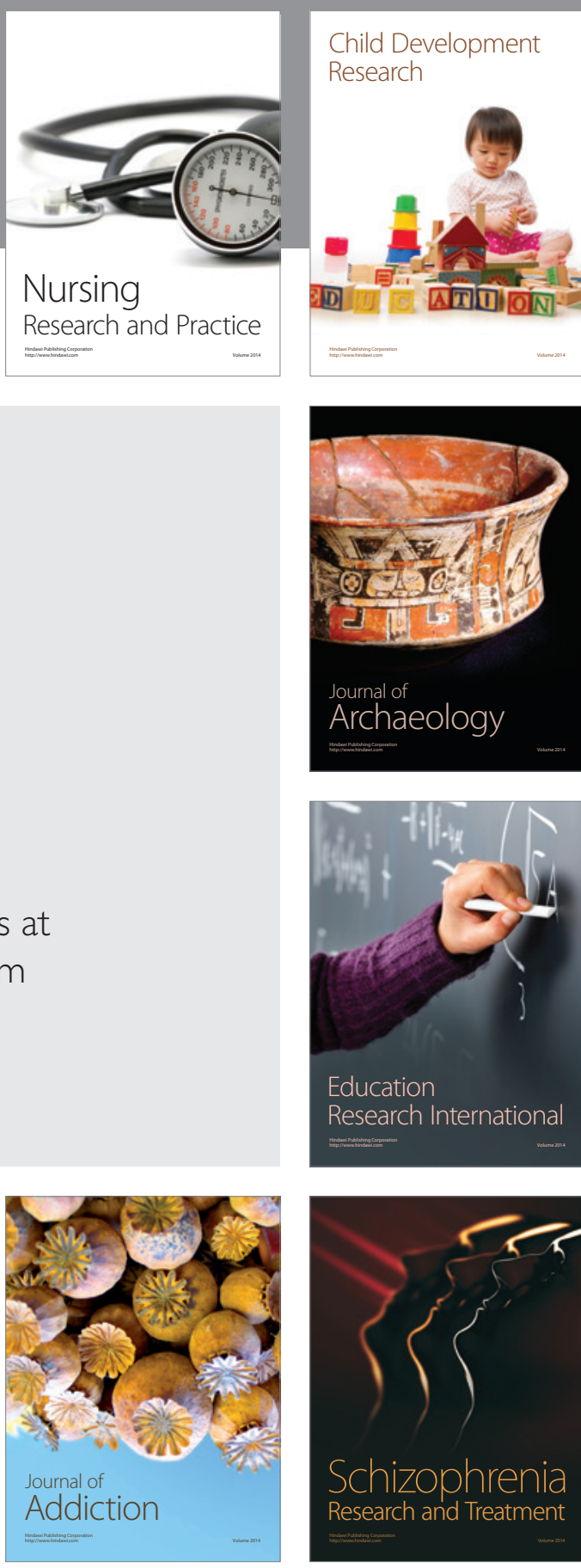

(D)
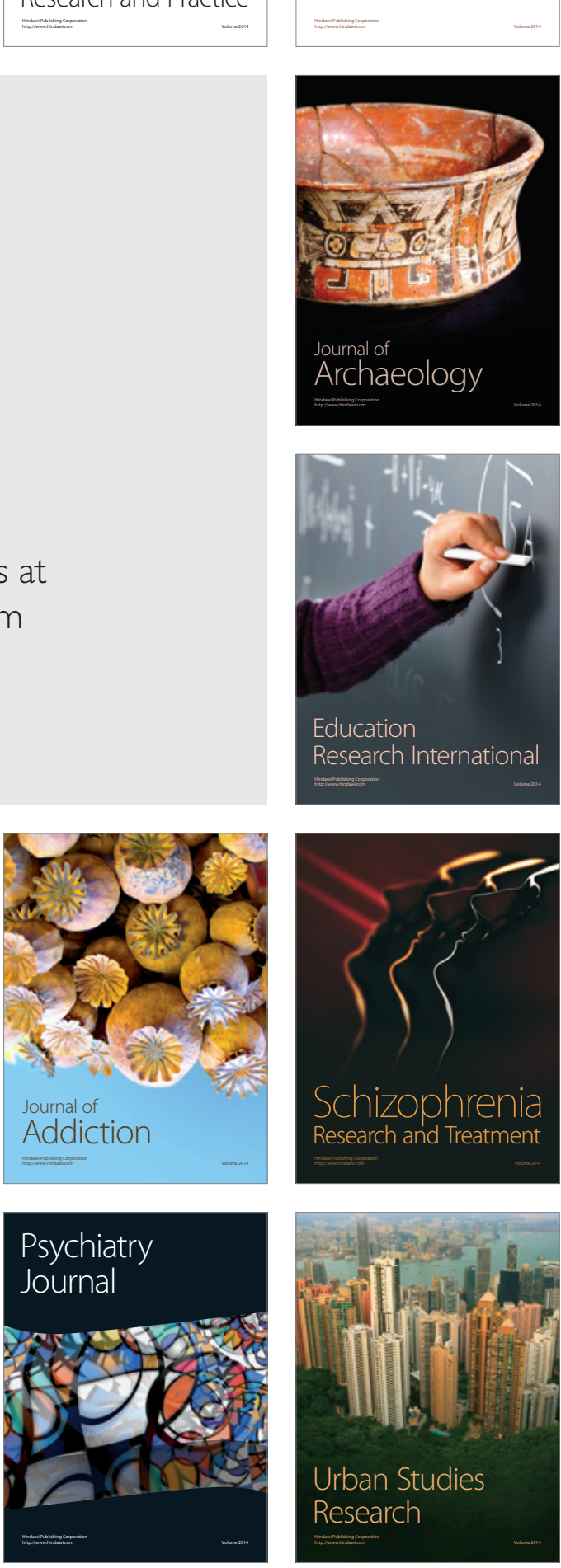